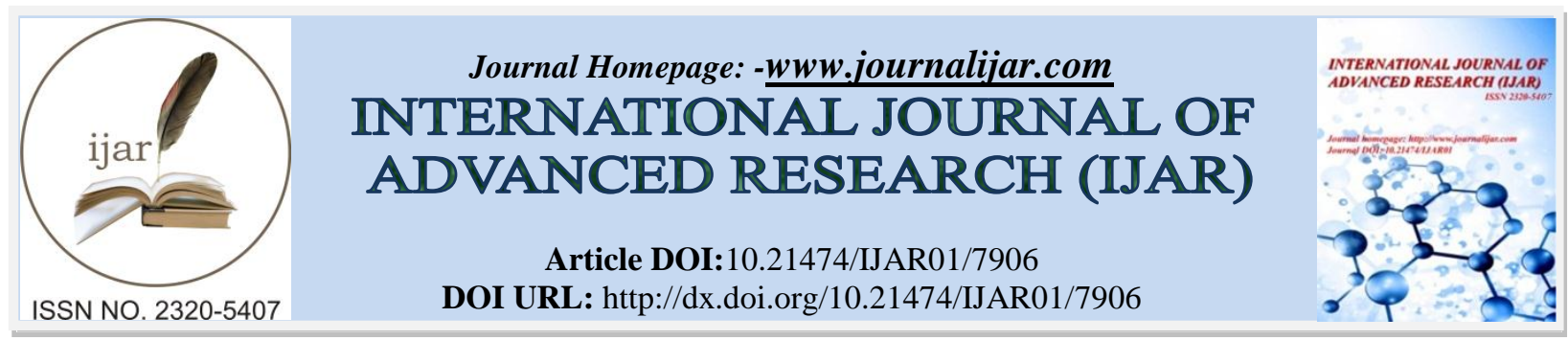

RESEARCH ARTICLE

\title{
COMPLICATIONS OCULAIRES DE LA PORPHYRIE CUTANEE TARDIVE : A PROPOS D'UN CASOCULAR COMPLICATIONS IN PORPHYRIA CUTANEA TARDA : A CASE REPORT.
}

\section{LM. Hmidchat ${ }^{1}$, G. Abdellaoui ${ }^{2}$ and O. Cherkaoui ${ }^{2}$.}

1. Service d'ophtalmologie A, hôpital des spécialités, CHU de Rabat.

2. Université Mohammed V - Faculté de Médecine et de Pharmacie de Rabat.

\section{Manuscript Info}

Manuscript History

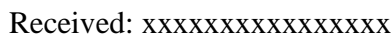

Final Accepted: $x x x x x x x x x x x$

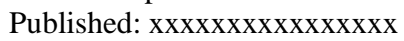

Keywords:

porphyrie cutanée tardive, atteinte oculaire, diagnostic, traitement.

\section{Abstract}

La porphyrie cutanée tardive est très peu connue des ophtalmologistes, les complications oculaires graves étant peu décrites dans la littérature. Nous rapportons le cas d'un patient porteur de porphyrie cutanée tardive, présentant une atteinte cornéenne avec une sclérite nécrosante bilatérale. Par l'étude de son évolution sous traitement et à la lumière des données de la littérature, nous tenterons de faire une mise au point sur la prise en charge diagnostique et thérapeutique de la porphyrie cutanée tardive et de ses atteintes oculaires.

Copy Right, IJAR, 2018,. All rights reserved.

\section{Introduction:-}

Les porphyries cutanées sont un groupe d'affections métaboliques rares secondaires à un déficit congénital ou acquis en une des huit enzymes impliquées dans la synthèse des porphyrines, substances qui interviennent dans la biosynthèse de l'hème.

Des mutations dominantes ou récessives d'un des gènes codant pour ces enzymes induisent une accumulation et une excrétion quantifiables des porphyrines et/ou de leurs précurseurs.

Les porphyries ont en commun des manifestations cutanées, dont la principale est une photosensibilité aiguë ou subaiguë.

Les manifestations ophtalmologiques sont quant à elles très rares, mais peuvent être graves et cécitantes. Nous présentons un cas de porphyrie cutanée tardive, avec atteinte cornéenne et nécrose scléro-conjonctivale bilatérale.

\section{Cas clinique}

Nous rapportons le cas d'un patient âgé de 42 ans, suivi depuis l'âge de 20 ans en dermatologie pour porphyrie cutanée tardive sporadique, biologiquement prouvée. Il a été hospitalisé en urgence dans notre service pour un abcès de cornée sévère au niveau de l'œil gauche.

L'examen ophtalmologique à son admission révèle au niveau de l'œil gauche un abcès de cornée para-central temporal prenant toute l'épaisseur cornéenne, avec hypopion (figure 1). 


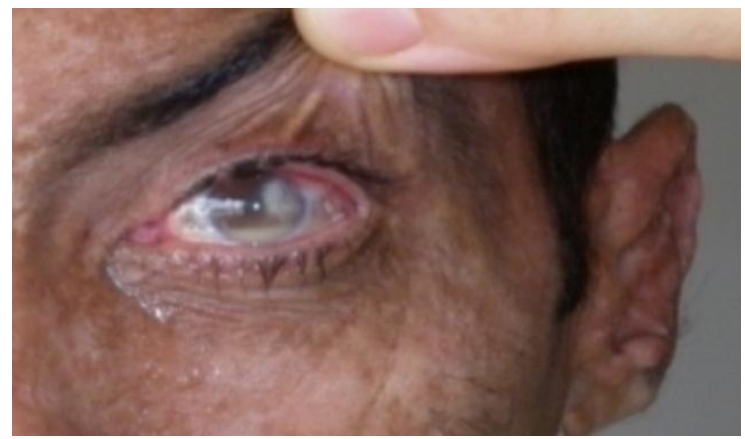

Figure 1:-Abcès à hypopion de l'œil gauche.

L'examen des deux yeux retrouve par ailleurs plusieurs zones d'amincissement et de nécrose scléro-conjonctivale (figure 2), présentant les caractéristiques suivantes : de profondeur variable, situées en zone interpalpébrale, exposée à la lumière (méridien de 3h-9h), proches du limbe, surmontées de plaques jaunâtres épaisses, enchâssées dans la sclère par endroits.
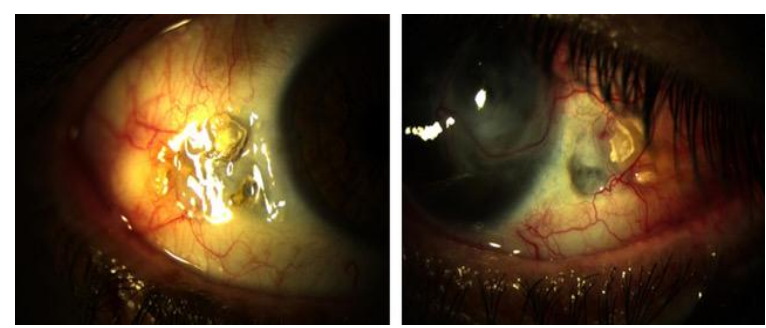

Figure 2:-Plaques+ Zones De Nécrose Scléro-Conjonctivale En Odg.

L'examen général retrouve des lésions dermatologiques évocatrices de porphyrie cutanée : une hyperpigmentation cutanée, une photosensibilité avec éruptions bulleuses, des cloques, une hypertrichose. L'ensemble de ces lésions prédominant sur le visage et au dos des mains (figure 3).
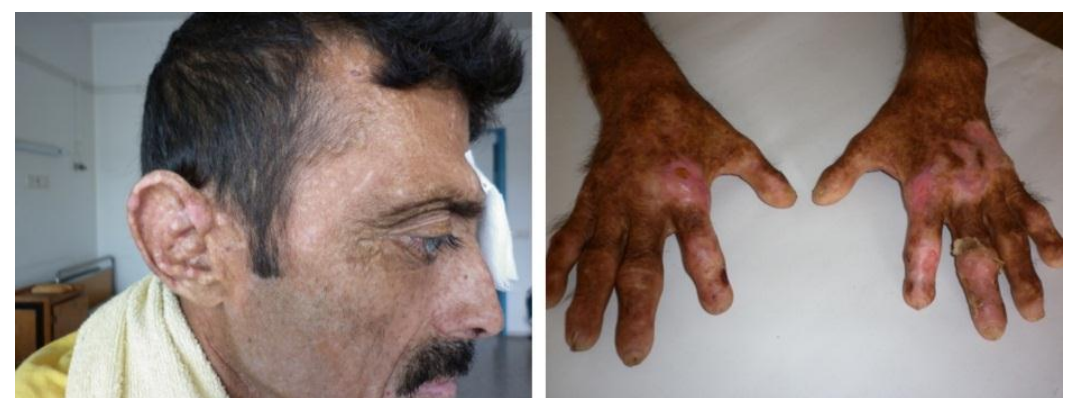

Figure 3:-tâches d'hyperpigmentation+ photosensibilisation au niveau du visage avec bulles, cloques et hypertrichose au niveau des mains.

Des prélèvements cornéens à visée diagnostique microbiologique ont été réalisés en urgence et le patient a était mis sous collyres antibiotiques renforcés horaires après une dose de charge, associés à la fluoroquinolone par voie orale. L'antibiothérapie a été adapté après isolement du staphylocoque épidermidis.

Un bilan biologique a parallèlement été réalisé:

1. Dosage des uroporphyrines urinaires, et des coproporphyrines : très élevées, avec un rapport >3

2. Dosage du fer sérique, de la ferritinémie, et bilan hépatique complet: sans anomalies.

3. L'analyse anatomopathologique d'une plaque sclérale prélevée en temporal au niveau de l'oeil droit a retrouvé un tissu nécrotique, calcifié par endroits (figure 4 et 5). 


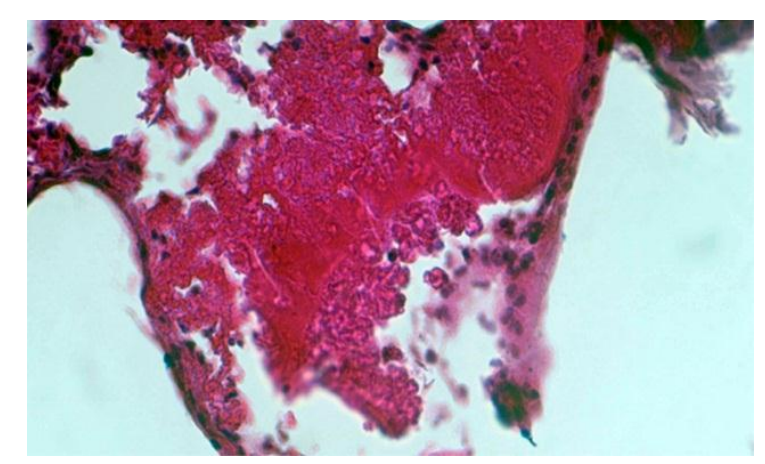

Figure 4:-Examen anatomopathologique de la plaque prélevée montrant un épithélium pavimenteux stratifié surmontant un tissu nécrotico-hémorragique.

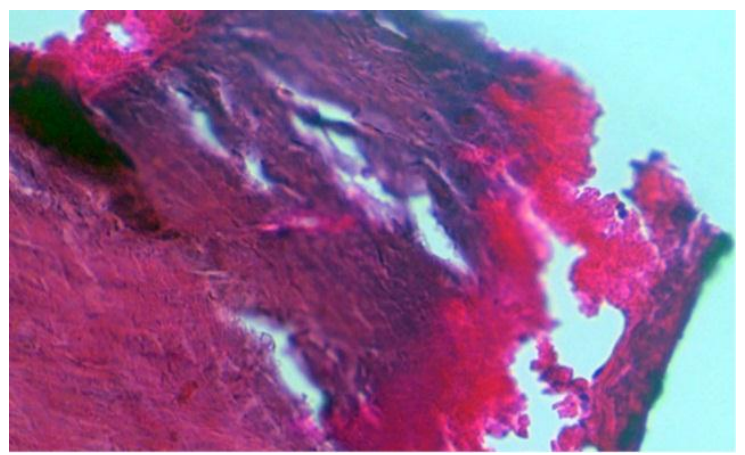

Figure 5:-Aspect anatomopathologique de nécrose calcifiée.

Une enquête familiale a été réalisée retrouvant un frère présentant une scléromalacie bilatérale (figure 6), avec sur le plan général une anémie hémolytique en cours d'exploration, ainsi qu'une sœur porteuse de lésions dermatologiques à type d'hyperpigmentation cutanée, et dont l'examen ophtalmologique retrouve uniquement un pinguecula bilatéral (figure 7).

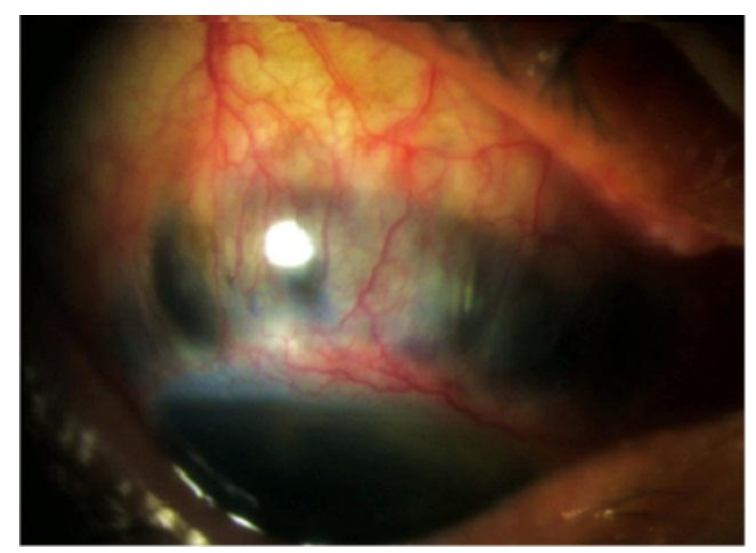

Figure 6:-Scléromalacie en supérieur chez le frère. 


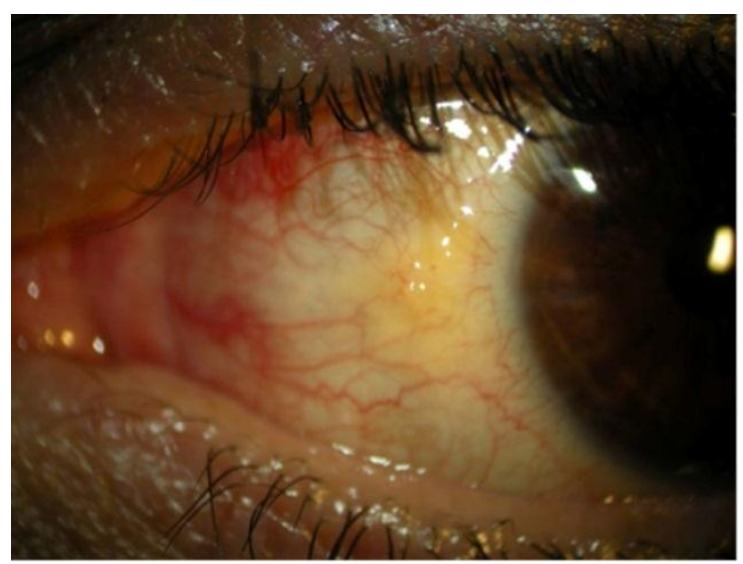

Figure 7:-Pinguecula en nasal chez la soeur

En collaboration avec les dermatologues un traitement de fond par antipaludéens de synthèse (APS) a été mis en route : chloroquine $200 \mathrm{mg}$, deux fois par semaine, associée à lavage oculaire régulier par du sérum physiologique, l'instillation d'agents mouillants et une photo-protection stricte.

Le traitement a permis l'amendement de l'infection, une cicatrisation très lente des zones de nécrose sclérale. Il n'y a pas eu de nouveau signe infectieux ou inflammatoire oculaire.

L'évolution à long terme a en revanche été grevée de complications: amincissement cornéen important de l'œil gauche avec descemétocèle (figure 8).

Sur le plan dermatologique, il y a eu une régression partielle des lésions cutanées.

Après un recul de 32 mois, le bilan sanguin de contrôle a montré une diminution progressive des porphyrines.

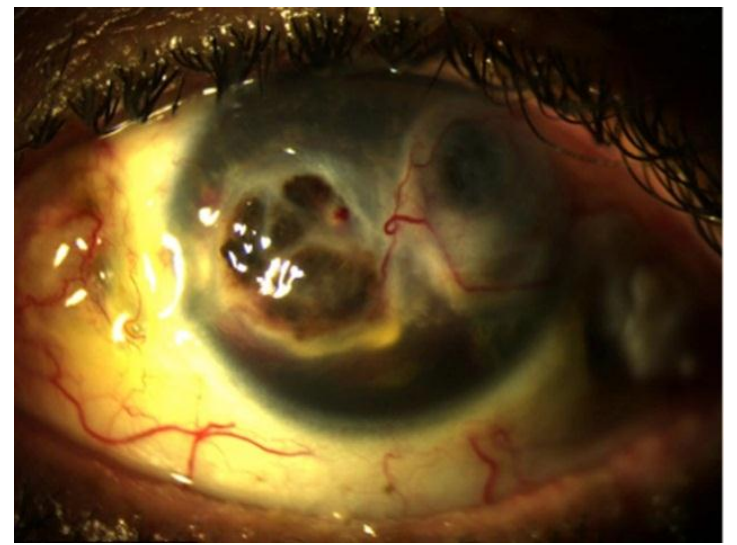

Figure 8:-Amincissement scléro-conjonctival, descemétocèle de l'œil gauche.

\section{Discussion:-}

La porphyrie cutanée tardive occupe une place majeure parmi les différents types de porphyries, c'est la seule affection pour laquelle on distingue une forme congénitale (PCT héréditaire ou PCT de type II) et une forme acquise (PCT sporadique ou PCT de type I). Elle est liée à un déficit en une enzyme impliquée dans la synthèse de l'hème: l'uroporphyrinogène décarboxylase conduisant à une accumulation de l'URO I porphyrine dans le sang et les urines [1]. C'est la porphyrie cutanée la plus fréquente de l'adulte.

Ses manifestations cliniques sont principalement dermatologiques et s'expliquent par la forte réactivité des porphyrines aux rayons lumineux. En effet, ces métabolites accumulés au niveau de la peau ainsi que d'autres zones photo-exposées (dont notamment la surface oculaire) vont absorber la lumière de certaines longueurs d'ondes (400 à $410 \mathrm{~nm}$ ) [2] et générer ainsi des radicaux libres hautement réactifs, à l'origine des lésions tissulaires photo-induites. 
Les manifestations ophtalmologiques de la PCT sont rares et méconnues. Elles sont dominées par les nécroses sclérales ainsi que par les ulcères cornéens et conjonctivaux.

Dans leur étude, Takamura et al ont démontré la présence de porphyrines dans les larmes [3]. Les porphyrines entraînent la formation de vésicules au niveau des régions exposées de la paupière, de la conjonctive, de la cornée et de la sclère.

La conjonctive peut présenter des lésions ptérygoïdes, des phlyctènes et des ulcérations inflammatoires [2] qui évoluent par la suite vers la fibrose et les symblépharons, réalisant un tableau de conjonctivite fibrosante [4]. Il en résulte une déshydratation de la sclère, à l'origine d'une nécrose sclérale avec visibilité anormale du tractus uvéal sous-jacent. Ceci est parfaitement illustré par les images prises de notre patient. Sur le plan histologique, les marges de la zone nécrotique sont souvent hyperplasiques, avec présence de fibrose interstitielle et de dégénérescence collagène induite par une infiltration modérée de cellules inflammatoires [2].

Par la suite, la cornée peut présenter des ulcères de profondeur variable, pouvant être sujets à un retard de cicatrisation ou à une surinfection microbienne, comme cela a été le cas chez notre patient. Les complications sont généralement de mauvais pronostic, tant sur le plan anatomique (perforation) que fonctionnel (optique): descemétocèle, perforation, surinfection polymicrobienne, cicatrice fibreuse stromale, astigmatisme, néovaisseaux [5].

De façon exceptionnelle, ont été décrits des cas d'atteinte rétinienne avec alternance de zones d'atrophie et de zones pigmentées [6].

Si l'on évoque une porphyrie, la conduite diagnostique associe un interrogatoire minutieux à la recherche d'antécédents familiaux, un examen clinique complet (en particulier dermatologique et neurologique), et des dosages biochimiques des porphyrines et de leurs précurseurs dans les urines, les selles et le sang.

Dans des laboratoires spécialisés, il est possible de réaliser des mesures des activités enzymatiques spécifiques dans les fibroblastes ou les lymphocytes des patients.

Plus récemment, grâce à la biologie moléculaire, il est possible de réaliser un séquençage direct de l'ADN par PCR, permettant non seulement de confirmer le diagnostic, mais aussi de mieux comprendre les gènes impliqués et leur fonction, en vue d'améliorer le conseil génétique des malades et de leurs familles [1].

Comme les manifestations cliniques des porphyries sont photo induites, la première mesure préventive dans cette pathologie est la protection solaire: lunettes, chapeaux, écrans solaires, vêtements, gants.

En outre, l'instillation fréquente d'agents mouillants permet l'élimination par effet mécanique de "lavage" des porphyrines accumulées sur la surface oculaire, entraînant ainsi la cicatrisation progressive des nécroses sclérales. L'éviction des facteurs déclenchants (alcool, œstrogènes, médicaments porphyrinogéniques) et la prévention des hépatites $\mathrm{A}$ et $\mathrm{B}$ par la vaccination sont par ailleurs des mesures préventives indispensables à la gestion de ce type de pathologies [7].

La prise en charge des complications oculaires dépend du type de manifestations locales.

Ainsi, en présence de nécrose sclérale pré-perforante, des greffes de membrane amniotique peuvent être proposées, avec toutefois un risque de récidive de nécrose sur le greffon. Un patch scléral s'avère parfois indispensable afin de restaurer l'intégrité du globe en cas de perforation sclérale avérée (5). Certains auteurs proposent des greffes allogéniques limbiques cornéo-sclérales, permettant la prolifération et la migration des cellules souches limbiques, ayant en outre un effet immunosuppresseur protégeant le greffon des réactions immunologiques (8).

La prise en charge des manifestations cornéennes dépend aussi du type d'atteinte: greffe de membrane amniotique en cas d'ulcère creusant ou présentant un retard de cicatrisation, colle cyano-acrylate, voire parfois greffe de cornée transfixiante en urgence en cas de perforation de grand diamètre. Plus récemment, certaines équipes ont proposé la mise en place de kératoprothèses, avec des résultats à moyen et long terme très encourageants (9). 
Le traitement systémique de la maladie doit être associé et est dirigé habituellement par le dermatologue. Singal et al ont reporté les résultats d'une étude pilote visant à tracer les grandes lignes thérapeutiques actuelles [10].

Ainsi, le traitement repose essentiellement sur les phlébotomies répétées : une unité de sang (jusqu'à $500 \mathrm{~mL}$ ) peut être retirée approximativement toutes les deux semaines. Elles ont pour but de diminuer progressivement la surcharge ferrique hépatique et doivent être arrêtées lorsque la ferritinémie est abaissée en-deçà du seuil de 20 $\mathrm{ng} / \mathrm{mL}$. Un contrôle régulier du taux d'hémoglobine ou de l'hématocrite doit être réalisé afin de dépister à temps une éventuelle anémie, contre-indication formelle à la poursuite des phlébotomies.

L'alternative thérapeutique est l'administration d'antipaludéens de synthèse (chloroquine ou hydroxy-chloroquine) à faibles doses et au long cours. Leur but est l'élimination des porphyrines présentes en excès dans l'organisme. Il est classiquement recommandé d'espacer les prises de 3 à 4 jours, et ce pendant plusieurs mois. L'utilisation des APS dans cette indication serait à l'origine de rémissions cliniques et biochimiques comparables à celles obtenues grâce aux phlébotomies régulières [10].

L'arrêt des APS est préconisé dès la normalisation du taux de porphyrines sériques $(\leq 0.9 \mu \mathrm{g} / \mathrm{dL})$ sous traitement continu pendant au moins un mois. Singal et al ont obtenu des rémissions biochimiques en moyenne après 6 à 7 mois de traitement. La rémission clinique est habituellement plus tardive. Les patients doivent alors être prévenus de la nécessité de continuer à se protéger des rayonnements solaires. Dans notre cas, l'administration de chloroquine à raison de $200 \mathrm{mg}$, deux fois par semaine, a permis la stabilisation des lésions ophtalmologiques, la régression partielle des lésions cutanées, ainsi qu'une diminution progressive des porphyrines sériques à long terme.

\section{Références:-}

1. Poblet-Gutiérrez $\mathrm{P}$, Wiederholt $\mathrm{T}$, Gardlo K. Les porphyries: de la clinique à la génétique moléculaire. Ann Dermatol Venereol 2004;131:825 8.

2. Hammer $\mathrm{H}$, Korom I. Photodamage of the conjunctiva in patients with porphyria cutanea tarda. $\mathrm{Br} \mathbf{J}$ Ophthalmol 1992;76(10):592 3.

3. Takamura N, Kurihara K, Yamashita S, KondoM. Need for measurement of porphyrins in teardrops in patients with congenital erythropoietic porphyria. Br J Ophthalmol 2002;86(10):1188.

4. Park AJ, Webster GF, Penne RB, Raber IM. Porphyria cutanea tarda presenting as cicatricial conjunctivitis. Am J Ophthalmol 2002;134(4):619 21.

5. Sati A, Sangwan VS, Basu S. Porphyria: varied ocular manifestations and management. BMJ Case Rep 2013 May 22;2013.

6. Denton WJ, Jordan CW, McGill WJ. Bilateral, geographic, peripapillary, chorioretinal atrophy in a patient with porphyria cutanea tarda and high iron stores. Optometry 2011;82(10):632 41.

7. Balwani M, Desnick RJ. The porphyrias: advances in diagnosis and treatment. Blood 2012;120(23):4496 504.

8. Yan F, Lu Y, Yin J, Jiang F, Huang ZP. Allogeneic corneoscleral limbus tissue transplantation for treatment of the necrosis in porphyria eye disease. Int J Ophthalmol 2014; 7(4): 731733.

9. Sati A, Sangwan VS, Basu S, Kalaiselvan P. Boston keratoprosthesis for visual rehabilitation in porphyria cutanea tarda. BMJ Case Rep 2013 Feb 1;2013

10. Singal AK, Kormos-Hallberg C, Lee C, Sadagoparamanujam VM, Grady JJ, Freeman DH Jr et al. Low-dose hydroxychloroquine is as effective as phlebotomy in treatment of patients with porphyria cutanea tarda. Clin Gastroenterol Hepatol 2012;10(12):1402 9. 